\title{
Adenocarcinoma de yeyuno proximal: a propósito de un caso
}

\section{Proximal jejunal adenocarcinoma, a case report}

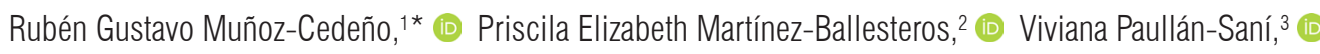 \\ Michelle Carolina Ricaurte-Enríquez, ${ }^{4}$ (b) Gema Nathalye Rodríguez-Chica. ${ }^{5}$
}

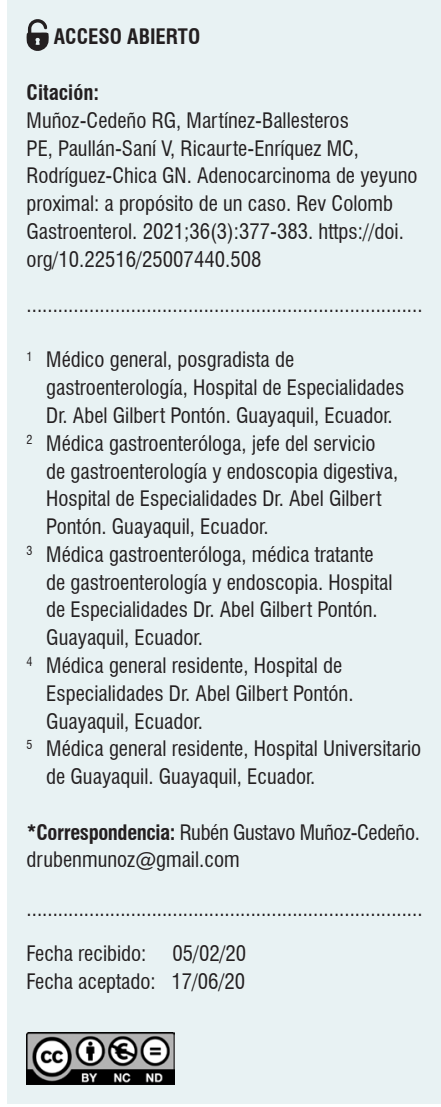

\begin{abstract}
Resumen
El adenocarcinoma de yeyuno es el tumor primario del intestino delgado más infrecuente y raro, y representa el $3 \%$ de todos los cánceres gastrointestinales, con una incidencia anual estimada de 0,3 a 2 casos por cada 100000 personas. En Estados Unidos y España representa el 0,4 \% de los cánceres gastrointestinales y el $0,2 \%$ de las muertes asociadas a malignidad, con síntomas inespecíficos como anemia de etiología inexplicable, dolor abdominal y pérdida de peso; la mayoría de ellos son diagnosticados en etapas avanzadas de la enfermedad. A continuación, se presenta el caso de una paciente femenina de 46 años con síntomas inespecíficos, pérdida de peso, dolor abdominal y vómito, cuya tomografía abdominal computarizada evidenció el sitio y extensión de la lesión neoplásica, mientras que la cápsula endoscopia y enteroscopia mostró una lesión neoplásica en el yeyuno proximal. La histopatología reportó un adenocarcinoma de yeyuno moderadamente diferenciado que presentó resultados favorables gracias a la resección quirúrgica curativa, lo cual mejoró el pronóstico de vida a 5 años y tuvo un seguimiento favorable hasta la actualidad.
\end{abstract}

\section{Palabras clave}

Tumor de yeyuno, adenocarcinoma de yeyuno, neoplasias de intestino delgado.

\section{Abstract}

Jejunal adenocarcinoma is the rarest primary small bowel tumor and represents $3 \%$ of all gastrointestinal cancers, with an estimated annual incidence of 0.3 to 2 cases per 100000 people. This disease accounts for $0.4 \%$ of gastrointestinal cancers and $0.2 \%$ of deaths associated with malignancy in the United States and Spain, with nonspecific symptoms, such as anemia of unexplained etiology, abdominal pain, and weight loss; most cases are diagnosed in advanced stages of the disease. The following is the case of a 46-year-old female with non-specific symptoms, weight loss, abdominal pain and vomiting. A computerized tomography scan of the abdomen allowed determining the site and extension of the tumor, while a capsule endoscopy and an enteroscopy showed a neoplastic lesion in the proximal jejunum. A histopathology analysis reported moderately differentiated adenocarcinoma of jejunum with favorable results after a curative surgical resection that improved the prognosis of life at 5 years and with favorable follow-up until to date.

\section{Keywords}

Jejunum tumor; Jejunum adenocarcinoma; Small bowel neoplasms.

\section{INTRODUCCIÓN}

Los tumores de intestino delgado son infrecuentes y representan solo $3 \%-6 \%$ de las neoplasias gastrointestinales. La incidencia en España es baja, de 0,4 a 1 por cada 100000 habitantes/año: $2 / 3$ son malignos y solo $1 / 3$ son benig$\operatorname{nos}^{(1)}$. En Estados Unidos la incidencia anual estimada es de 0,3 a 2 casos por cada 100000 personas, lo que repre- 
senta el 0,4\% de los cánceres gastrointestinales y el 0,2 \% de las muertes asociadas a malignidad, con tasas de prevalencias más altas en la población negra que en la blanca; esto ha aumentado recientemente. En Panamá se estima menos de $1 \%$ y entra dentro del grupo de causas de malignidad en general ${ }^{(2)}$. En México, un registro histopatológico de neoplasia malignas en 2006 registró un total de 106238 tumores malignos, donde solo $0,32 \%$ se localizaron en el intestino delgado ${ }^{(3)}$. En Ecuador no existen datos sobre tumores de intestino delgado.

No se sabe mucho de su historia natural; la mayor incidencia se da después de los 60-70 años. Según datos del Surveillance, Epidemiology, and End Results (SEER) Program de 1992-2006, la tasa de incidencia de hombres y mujeres es de 1,45 y 1,00 , respectivamente, y en la raza negra su presentación es inespecífica, asintomático en etapas tempranas por largos períodos de tiempo y conforme progresa la enfermedad se desarrollan los síntomas, por lo que existe un retraso en el diagnóstico durante, aproximadamente, 6 a 8 meses. El tratamiento quirúrgico está limitado al tamaño de la lesión y al grado de invasión ${ }^{(4)}$.

\section{PRESENTACIÓN DEL CASO}

Se trata de una paciente femenina de 46 años de edad, de raza mestiza, sin antecedentes patológicos de importancia y con antecedentes quirúrgicos de histerectomía hace 8 años por miomas uterinos, quien presenta un cuadro clínico de inicio de 3 meses de evolución por un dolor abdominal generalizado. En la escala visual análoga (EVA) el dolor es de $6 / 10$ puntos, el cual está acompañado de vómitos de tipo alimenticio 2 a 3 al día y pérdida de peso de aproximadamente $15 \mathrm{~kg}$. La paciente niega la presencia de alza térmica (fiebre) y sangrado digestivo. Refiere haber acudido con un médico particular, donde fue valorada por un dolor abdominal inespecífico, por lo que le realizaron una ecografía abdominal en la que encontraron una masa de aproximadamente $78 \times 68 \mathrm{~mm}$ entre el epigastrio e hipocondrio izquierdo. Por esta razón, la paciente acude a esta casa de salud y es valorada en el servicio de consulta externa con exámenes de ingreso, que reportan: hemoglobina de 10,20 $\mathrm{mg} / \mathrm{dL}$, hematocrito de $33,10 \%$, leucocitos $6300 / \mathrm{mm}^{3}$, plaquetas 631000 , marcadores tumorales negativos, sangre oculta positiva, albúmina $2 \mathrm{mg} / \mathrm{dL}$, proteínas totales 3,90 $\mathrm{mg} / \mathrm{dL}$. Con base en esto, se indicó la realización de estudios endoscópicos en busca de una lesión neoplásica a descartar. Al realizar la endoscopia digestiva alta se visualizó la presencia de una compresión extrínseca a nivel del cuerpo distal con gastropatía eritematosa en antro, mientras que la ileocolonoscopia resultó normal (Figura 1).

Además, se realiza una tomografía de abdomen y pelvis con doble contraste donde se presenta una masa circunferencial a nivel del yeyuno proximal, que permite el paso del medio de contraste y respeta los planos de clivajes en estadio I (Figuras 2 y 3 ). Se realiza un procedimiento con una cápsula endoscópica, la cual queda retenida a nivel del yeyuno, que permite observar una lesión elevada exofítica en el yeyuno proximal (Figura 4). Luego se procede a realizar una enteroscopia anterógrada a nivel del yeyuno proximal, donde se observa una lesión elevada, exofítica, ulcerada, friable, que ocupa más del $90 \%$ de la circunferencia, pero permite el paso del equipo hasta la región distal (Figuras 5 y 6). Desde allí se visualiza la cápsula retenida y es retirada con una pinza de malla. Finalmente, se toma una biopsia, la cual reporta en el informe histopatológico un adenocarcinoma tubulopapilar moderadamente diferenciado del yeyuno proximal (Figura 7).

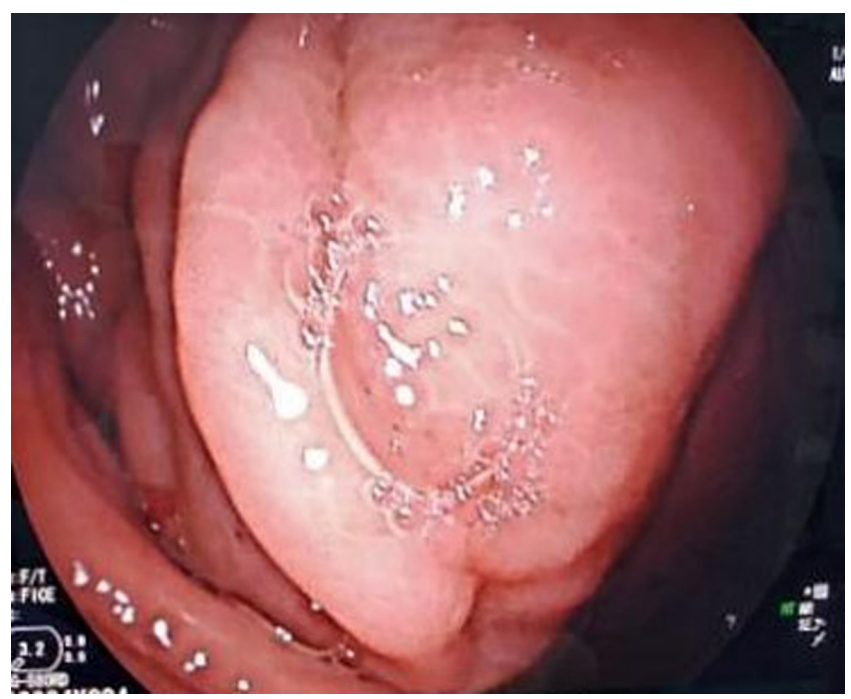

Figura 1. Videoendoscopia digestiva alta donde se observa una compresión extrínseca a nivel del cuerpo distal.

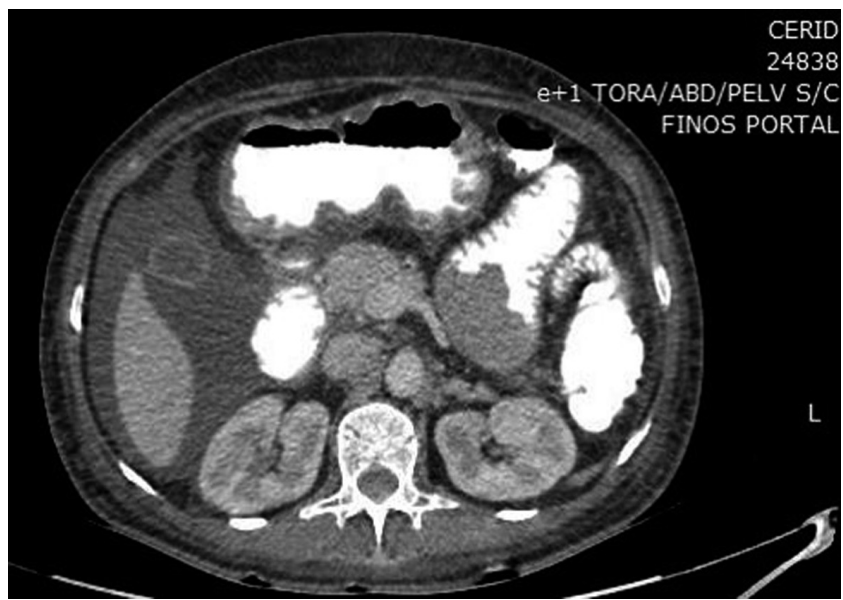

Figura 2. Tomografía computarizada de abdomen contrastada en el corte transversal muestra una masa circunferencial a nivel del yeyuno proximal. 


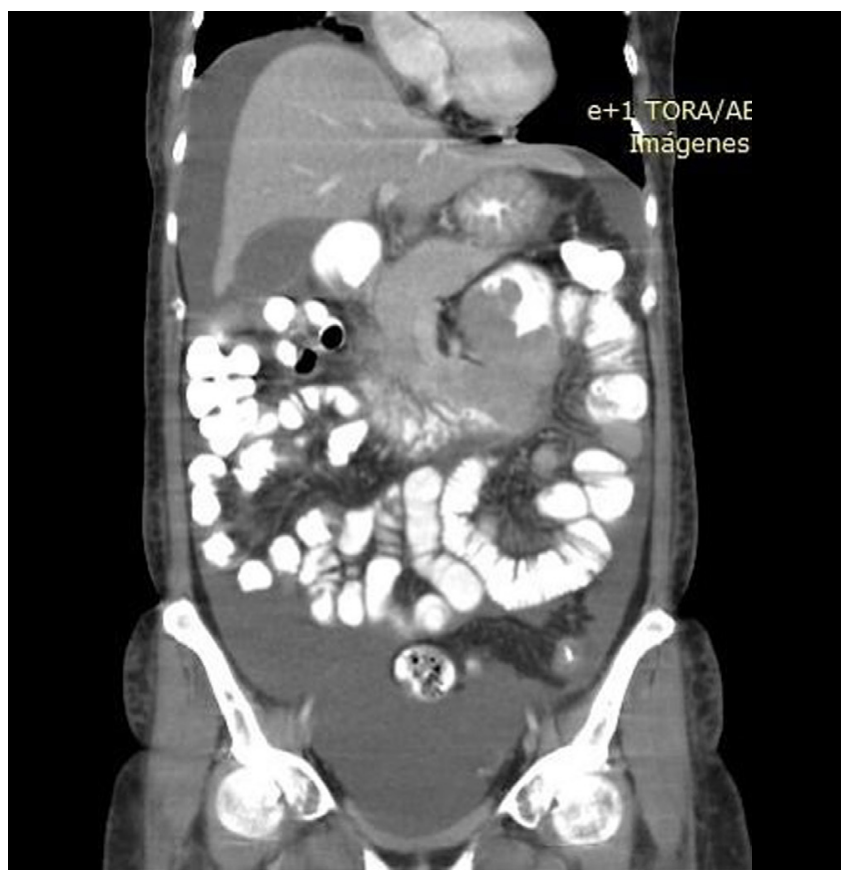

Figura 3. Tomografía computarizada de abdomen contrastada en el corte coronal muestra una masa circunferencial a nivel del yeyuno proximal.

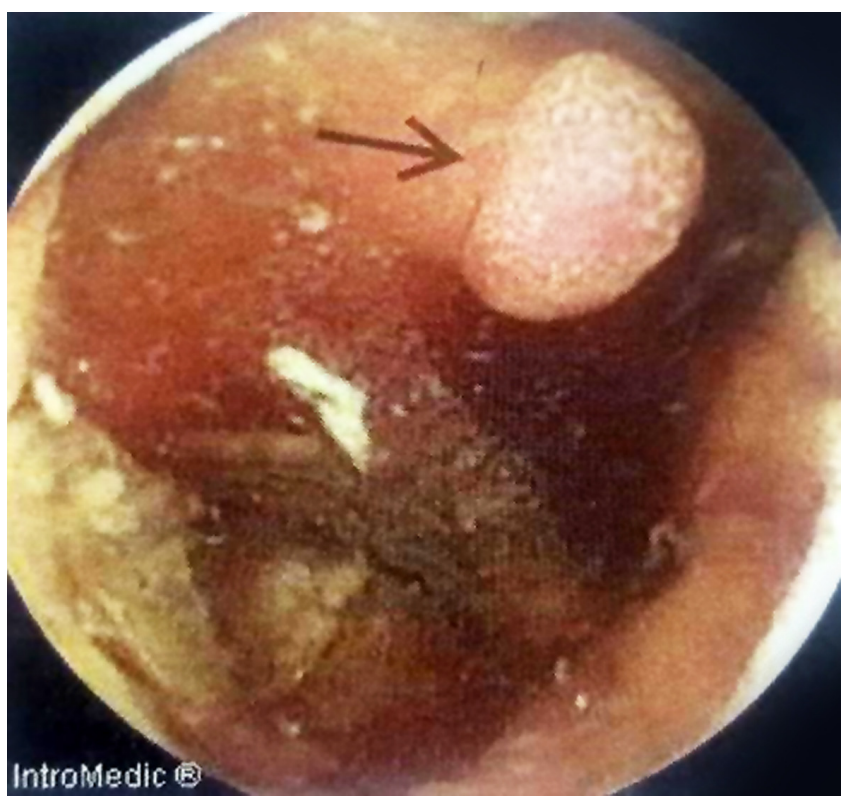

Figura 4. Video de la cápsula endoscópica que visualiza la presencia de una lesión elevada exofítica en el yeyuno.

La paciente es valorada por el servicio de cirugía, donde se le realiza una laparotomía exploratoria con resección intestinal tumoral del yeyuno de aproximadamente $15 \times 10$ $\mathrm{cm}$ (Figuras 8 y 9 ) más anastomosis terminolateral entre el duodeno y el yeyuno, sin signos de carcinomatosis peritoneal. El reporte histopatológico de la macrobiopsia es: tamaño tumoral de $11,5 \times 7 \times 3 \mathrm{~cm}$; tipo histopatológico: adenocarcinoma moderadamente diferenciado G2; patrón velloso, cuya extensión tumoral invade la muscularis propia; márgenes: bordes quirúrgicos más cercanos a la lesión distal a $5 \mathrm{~mm}$ por microscopia, borde quirúrgico más lejano de la lesión libre de neoplasia, borde quirúrgico radial libre de neoplasia, ganglios linfáticos con hiperplasia sinusoidal libres de neoplasia. Esto dio como resultado márgenes de $\mathrm{R} 0$ libres de lesión neoplásica.

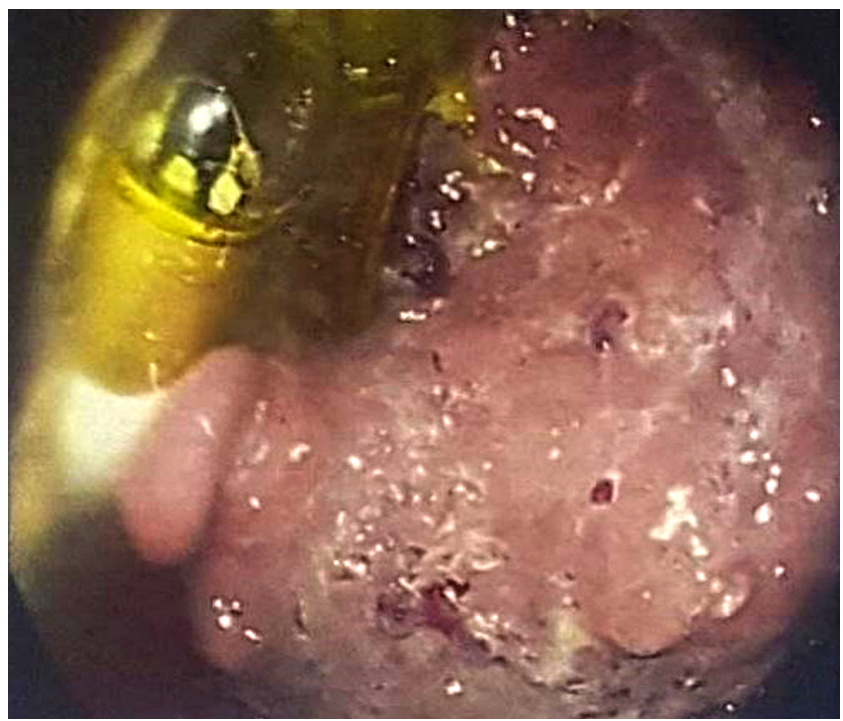

Figura 5. Enteroscopia en la que se observa una masa elevada, exofítica, ulcerada, friable, que ocupa más del $90 \%$ de la circunferencia y muestra la presencia de la cápsula endoscópica retenida.

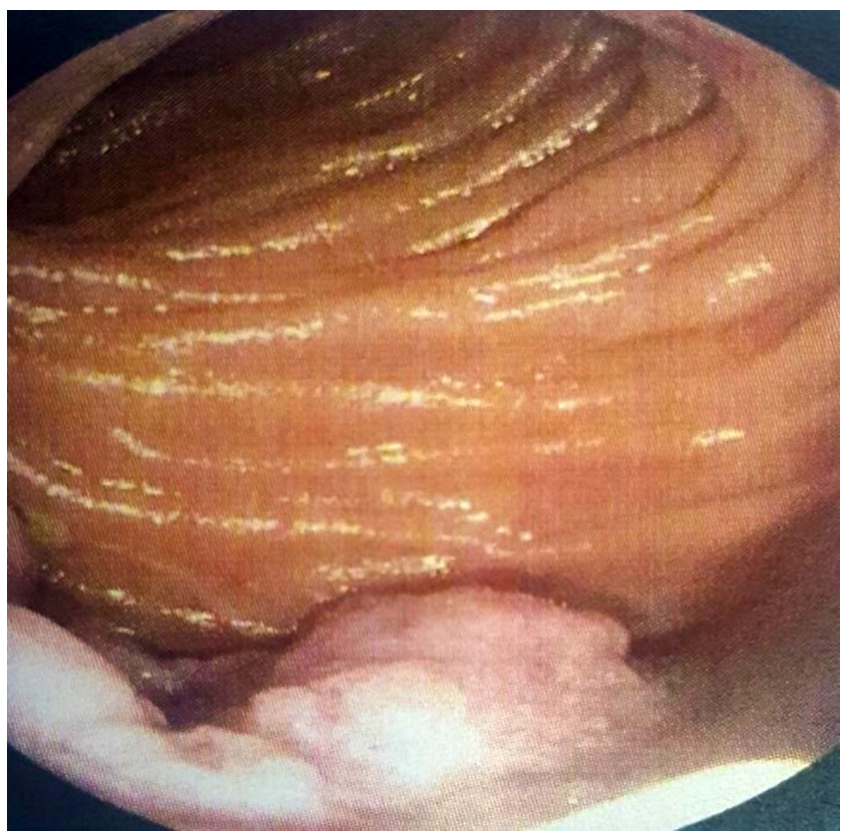

Figura 6. Enteroscopia que muestra la lesión elevada exofítica, la cual ocupa parte de la circunferencia intestinal del yeyuno. 


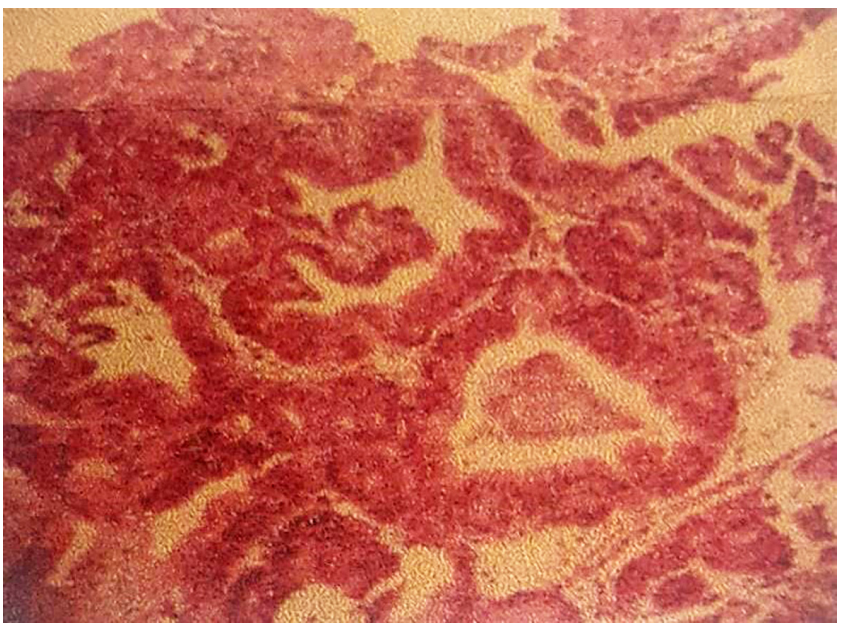

Figura 7. Histopatología adenocarcinoma moderadamente diferenciado.

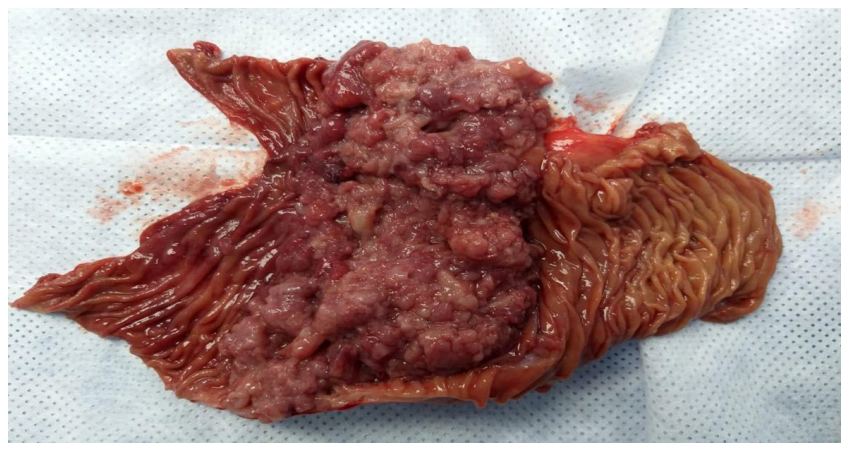

Figura 8. Aspecto macroscópico de la masa del yeyuno proximal.

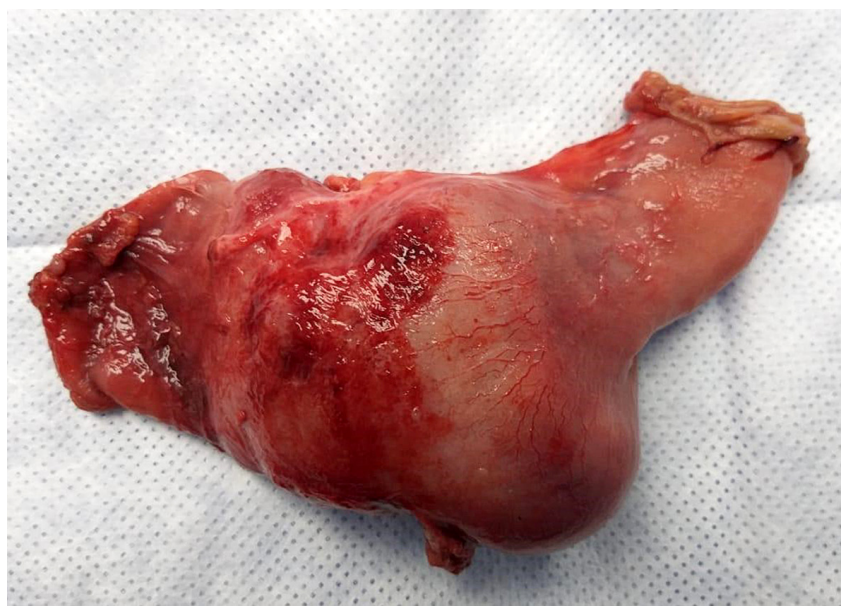

Figura 9. Aspecto macroscópico de la masa del yeyuno proximal.

Durante la hospitalización, la paciente fue tratada por el servicio de nutrición con soporte nutricional parenteral de $35 \mathrm{kcal} / \mathrm{kg} /$ día por 3 días, después con nutrición mixta con tolerancia oral hasta la progresión de la dieta sin presentar complicaciones posquirúrgicas $\mathrm{y}$, finalmente, fue dada de alta médica con seguimiento por consulta externa. Actualmente, la paciente está en seguimiento multidisciplinario por gastroenterología, cirugía, oncología, psicología y nutrición, y ha tenido una evolución favorable con ganancia de peso, indicaciones nutricionales y tomografía abdominal contrastada al año negativa.

\section{DISCUSIÓN}

El intestino delgado se conforma de tres partes: duodeno, yeyuno e íleon. En su totalidad, representa aproximadamente el $75 \%$ del sistema digestivo y mide alrededor de 15 a 20 pies de longitud, es decir de 6 a 8 metros. Solamente del $6 \%-25 \%$ de las neoplasias gastrointestinales se presentan en el intestino delgado. El yeyuno sigue al duodeno, encontrándose principalmente en la región umbilical del abdomen $^{(5)}$.

En el intestino delgado se pueden presentar cinco tipos de cáncer: adenocarcinomas, sarcomas, tumores estromales del tracto gastrointestinal (gastrointestinal stromal tumor, GIST), tumores neuroendocrinos y linfomas. El más común es el adenocarcinoma, que representa entre el $30 \%-40 \%$ de los mencionados. Se reporta que, de los adenocarcinomas, el $57 \%$ se encuentra en el duodeno, el $29 \%$ en el yeyuno y el $10 \%$ en el íleon ${ }^{(4,6)}$. Además, se ha mencionado que la bilis debería estudiarse como posible agente carcinogénico para explicar la prevalencia más alta de adenocarcinomas en el duodeno ${ }^{(7)}$. Se consideran factores de riesgo para lesiones malignas la enfermedad celíaca, la poliposis adenomatosa familiar, el síndrome de PeutzJeghers, la enfermedad de Crohn, entre otras patologías ${ }^{(8)}$.

La anatomía del yeyuno permite que sea el lugar donde se produce la mayor absorción de nutrientes, aminoácidos, vitaminas y minerales. Esta sección del intestino tiene válvulas conniventes, que hacen que su pared sea más gruesa que en el íleon. Dichas válvulas junto a las vellosidades representan la razón por la que la capacidad absortiva del yeyuno es alta. También contiene glándulas de Brunner, que son glándulas submucosas productoras de moco; sin embargo, las mismas se encuentran en mayor cantidad en el duodeno $^{(9)}$. Se ha reportado que aproximadamente el $80 \%$ de casos de adenocarcinomas yeyunales se desarrollan a partir de 50-60 cm del ligamento de Treitz ${ }^{(10)}$.

El adenocarcinoma de yeyuno inicialmente se puede presentar con síntomas inespecíficos, como molestias abdominales, dolor, distensión abdominal o náuseas que podrían confundirse con otras patologías, siendo finalmente el cuadro de presentación la obstrucción en el $40 \%$ de los casos o de hemorragia gastrointestinal en el $24 \%$ de los $\operatorname{casos}^{(4,11)}$. También es asintomático en etapas tempranas, lo que retrasa el diagnóstico durante aproximadamente 6 a 8 meses; sin embargo, a medida que progresa la enferme- 
dad, los síntomas se manifiestan con el dolor abdominal, la pérdida de peso (que son los síntomas más comunes), el sangrado gastrointestinal, vómitos, náuseas y obstrucción (que son los menos comunes). En el examen físico rara vez se puede encontrar una masa palpable en el abdomen y signos de irritación peritoneal, tal vez debido a obstrucción o perforación $^{(12)}$.

En los datos de los exámenes de laboratorio se puede evidenciar anemia leve debido a la pérdida de sangre crónica y de etiología no identificada; ante esto, siempre se debe sospechar de un proceso neoplásico. Las pruebas de función hepática pueden revelar hiperbilirrubinemia en caso de tumores duodenales y una elevación de las transaminasas cuando existen metástasis hepáticas ${ }^{(13)}$.

Para el diagnóstico es fundamental realizar el tránsito intestinal, ecografía abdominal, tomografía computarizada abdominal, enteroclisis por tomografía, enteroclisis por resonancia, endoscopia digestiva alta, enteroscopia anterógrada y cápsula endoscópica. El tránsito intestinal con bario es la prueba estándar para las lesiones intraluminales o anormalidades de la mucosa más allá del duodeno yeyunal, pero tiene una sensibilidad limitada (53\%-83\%) para los cánceres de intestino delgado. La tomografía computarizada abdominal con contraste oral e intravenoso revela el sitio exacto y la extensión de la enfermedad local, con realce periférico intenso por el contraste intravenoso, así como la presencia de metástasis hepáticas. No obstante, es deficiente en la detección de tumores pequeños primarios, con una especificidad y sensibilidad del $57 \%$; es útil para la estadificación, pero para las patologías del intestino delgado deben usarse otras modalidades de imagen ${ }^{(13,14)}$.

Para el estudio y la investigación de estas lesiones se usan la enteroclisis por tomografía y la enteroclisis por resonancia, ya que son más sensible ( $85 \%$-95\%), con una especificidad del $90 \%-96 \%$, pudiendo observar el tumor como una lesión anular estenosante de mínimo $3 \mathrm{~mm}$ de longitud ${ }^{(13)}$. La endoscopia digestiva alta no puede pasar más allá del ligamento de Treitz; aunque identifica alrededor del $93 \%$ de los tumores duodenales, en general la sensibilidad es solo del $31 \%^{(10)}$.

En la enteroscopia anterógrada solo se puede visualizar el $30 \%$ de la longitud del intestino delgado, y puede diagnosticar más casos de adenocarcinoma de intestino delgado debido a que la mayoría de los adenocarcinomas se encuentran en los $60 \mathrm{~cm}$ proximales del yeyuno. La enteroscopia tiene una sensibilidad del $100 \%$, pero una especificidad de solo el $45 \%$ en los diagnósticos de tumores de intestino delgado; el $60 \%$ de los tumores identificados adenocarcinomas ${ }^{(15)}$.

La tasa de diagnóstico de los tumores del intestino delgado va en aumento, debido al uso de métodos como la cápsula endoscópica, que es el método de diagnóstico más sensible y específico para la enfermedad del intestino delgado. La cápsula detecta el sangrado gastrointestinal oscuro, con el que se diagnostica la mayoría de estos tumores, y el $50 \%$ a $60 \%$ de ellos son malignos. Este método ha superado la baja sensibilidad de los estudios con bario.

En cuanto al tratamiento, la única opción curativa es la quirúrgica. Se recomienda la resección total de la lesión con un margen mayor a $2 \mathrm{~cm}$ de longitud junto a una linfadenectomía extendida ${ }^{(16)}$. Las Guías Francesas de 2016 recomiendan, además, la realización de una tomografía toracoabdominopélvica para descartar metástasis a distancia y una endoscopía alta y baja por si existen otros tumores sugestivos de alguna patología genética desencadenante ${ }^{(17)}$. La resección curativa de los tumores del intestino delgado se obtiene en alrededor del $50 \%$; por ejemplo, en una revisión reciente hecha por Agrawal y colaboradores se demostró que la resección curativa para adenocarcinoma fue del $54,5 \%$ de los casos, con una tasa de mortalidad posoperatoria del 3,6\% ${ }^{(18)}$.

Existen factores de mal pronóstico para los pacientes con adenocarcinomas que incluyen: sexo masculino, mayores de 55 años, metástasis a distancia, tumores mal diferenciados, tumores T4, entre otros. Sin embargo, son de peor pronóstico los tumores duodenales y/o ileales en comparación con los yeyunales ${ }^{(12,19)}$, y el pronóstico de los tumores del intestino delgado es malo. La tasa de supervivencia de los pacientes a 5 años depende del estadio del tumor: en estadio I es de $50 \%$ a $60 \%$; en estadio II, de $39 \%$ a $55 \%$, en estadio III, de $10 \%$ a $40 \%$, y estadio IV, de $3 \%$ a $5 \%{ }^{(19)}$. La mayoría de las series informan una supervivencia a 5 años de $15 \%$ a $35 \%$, misma que después de la cirugía curativa aumenta de $40 \%$ a $65 \%$ en la mayoría de estudios. En un estudio realizado por Halfdanarson y colaboradores, en 491 pacientes con adenocarcinomas en intestino delgado, demostró que la tasa de supervivencia a 5 años fue de $26 \%$ en tumores no resecados ${ }^{(20)}$. Por otro lado, en casos de tumores resecados se reporta una tasa de supervivencia a los 5 años de $40 \%-65 \%$, con tasa de mortalidad posoperatoria del 3,6\%.

Debido a la recurrencia de lesiones aun después de la cirugía, se recomiendan ciclos de quimioterapia como tratamiento coadyuvante ${ }^{(21)}$. El 5-fluorouracilo es el agente farmacológico más utilizado en la quimioterapia de pacientes que ya han sido intervenidos quirúrgicamente y presentaban enfermedad extensa ${ }^{(10,22)}$.

El seguimiento de los pacientes está dado por pruebas de imágenes como la tomografía por emisión de positrones y la tomografía computarizada con doble contraste después del tratamiento para determinar si este fue eficaz o existe recidiva dependiendo del estadio y el tratamiento realizado ${ }^{(24)}$. 


\section{CONCLUSIONES}

El adenocarcinoma de yeyuno es uno de los tumores de intestino delgado más infrecuentes y de difícil diagnóstico por sus síntomas inespecíficos. Es por ello por lo que el médico requiere un alto índice de sospecha, para así realizar una investigación temprana y evitar un retraso en el diagnóstico debido al mal pronóstico. La sobrevida de estos pacientes está relacionada con el estadio clínico de la enfermedad al momento del diagnóstico. En nuestro caso, ante la sospecha de lesión neoplásica del intestino delgado el diagnóstico se realizó mediante métodos como la tomografía abdominal con doble contraste, que nos reveló el sitio exacto y la extensión del tumor; la cápsula endoscó- pica, que nos ayudó con el tipo de lesión y el sitio; y la enteroscopia anterógrada con doble balón, que nos ayudó con el diagnóstico y la toma de biopsia. Gracias a lo anterior se pudo realizar la cirugía curativa para mejorar la supervivencia a 5 años de la paciente, como en nuestro caso.

\section{Conflicto de interés}

No existen conflictos de interés.

\section{Fuente de financiación}

Propias de los autores.

\section{REFERENCIAS}

1. Martínez MA, Mingol F, Vaqué J, Baquero R, Mirc J. Adenocarcinoma de yeyuno: una entidad de difícil diagnóstico. Cir Esp. 2008;83(4):212-9. https://doi.org/10.1016/S0009-739X(08)70550-5

2. Martínez OA, Bissot RA, Botello KM. Adenocarcinoma de yeyuno: infrecuente cáncer gastrointestinal. Rev Colomb Cir. 2018;33(4):421-27. https://doi.org/10.30944/20117582.89

3. Sánchez-Ramón A, Cerino-Palomino V, Medina-Franco H. Tumores de intestino delgado: experiencia en el Instituto Nacional de Ciencias Médicas y Nutrición 'Salvador Zubirán”. Rev Gastroenterol Mex. 2012;77(4):181-5. https://doi.org/10.1016/j.rgmx.2012.06.004

4. Li J, Wang Z, Liu N, Hao J, Xu X. Small bowel adenocarcinoma of the jejunum: a case report and literature review. World J Surg Oncol. 2016;14(1):177. https://doi.org/10.1186/s12957-016-0932-3

5. Samaiya A, Deo SS, Thulkar S, Hazarika S, Kumar S, Parida DK, Shukla NK. An unusual presentation of a malignant jejunal tumor and a different management strategy. World J Surg Oncol. 2005;3(1):3. https://doi.org/10.1186/1477-7819-3-3

6. Cancer. Net Editorial Board. Tumor del estroma gastrointestinal - GIST. [Website] 2018 [consultado el 16 de enero de 2020]. Disponible en: https://www.cancer.net/cancertypes/gastrointestinal-stromal-tumor-gist

7. Negri E, Bosetti C, La Vecchia C, Fioretti F, Conti E, Franceschi S. Risk factors for adenocarcinoma of the small intestine. Int J Cancer. 1999;82(2):171-4. https://doi.org/10.1002/(SICI)10970215(19990719)82:2<171::AID-IJC3>3.0.CO;2-T

8. Green PH, Jabri B. Celiac disease and other precursors to small-bowel malignancy. Gastroenterol Clin North Am. 2002;31(2):625-39. https://doi.org/10.1016/S0889-8553(02)00010-9
9. Gourevitch D. The Anatomy and Physiology of the Small Bowel. En: Fielding JWL, Hallissey MT (editores). Upper Gastrointestinal Surgery. London: Springer; 2005. p. 39-44. https://doi.org/10.1007/1-84628-066-4_3

10. Bano F. Primary Jejunal Adenocarcinoma. J. Surg. Pak. 2013;18(2):103-104.

11. Beltran MA, Cruces KS. Primary tumors of jejunum and ileum as a cause of intestinal obstruction: a case control study. Int J Surg. 2007;5(3):183-91. https://doi.org/10.1016/j.ijsu.2006.05.006

12. Young JI, Mongoue-Tchokote S, Wieghard N, Mori M, Vaccaro GM, Sheppard BC, Tsikitis VL. Treatment and Survival of Small-bowel Adenocarcinoma in the United States: A Comparison With Colon Cancer. Dis Colon Rectum. 2016;59(4):306-15. https://doi.org/10.1097/DCR.0000000000000562

13. Shah PP, Kothari S. Jejunal adenocarcinoma-a case report with review. Indian J Surg. 2013;75(Suppl 1):82-5. https://doi.org/10.1007/s12262-011-0354-y.

14. Aparicio T, Zaanan A, Svrcek M, Laurent-Puig P, Carrere N, Manfredi S, Locher C, Afchain P. Small bowel adenocarcinoma: epidemiology, risk factors, diagnosis and treatment. Dig Liver Dis. 2014;46(2):97-104. https://doi.org/10.1016/j.dld.2013.04.013

15. Krishnamurthy P, Varghese SE, Gopalswamy N, Hillman N, Ali SA. Small-bowel adenocarcinoma: case report and review of literature on diagnosis of small-bowel tumors. Gastroenterol Hepatol (N Y). 2007;3(2):129-35.

16. Rongviriyapanich A. Adenocarcinoma of jejunum. J Surg Case Rep. $2018 ; 2018(8)$ :rjy234. https://doi.org/10.1093/jscr/rjy234

17. Locher C, Afchain P, Carrere N, Samalin E. Adénocarcinome de l'intestin grêle. En: Thésaurus National de Cancérologie Digestive [internet]. Francia: Onconormandie; 2016 [consultado el 16 de enero de 
2020]. Disponible en: https://www.oncorif.fr/wp-content/uploads/2017/03/PR1_TNCD_22_01_Chap_13 Adenocarcinome_de_1_intestin_grele_2016.pdf

18. Agrawal S, McCarron EC, Gibbs JF, Nava HR, Wilding GE, Rajput A. Surgical management and outcome in primary adenocarcinoma of the small bowel. Ann Surg Oncol. 2007;14(8):2263-9.

https://doi.org/10.1245/s10434-007-9428-2

19. Ahmed N, Rana HN. Primary Jejunal adenocarcinoma - An unsuspected culprit. J Pak Med Assoc. 2018;68(3):471-474.

20. Halfdanarson TR, McWilliams RR, Donohue JH, Quevedo JF. A single-institution experience with 491 cases of small bowel adenocarcinoma. Am J Surg. 2010 Jun; 199(6):797-803. https://doi.org/10.1016/j.amjsurg.2009.05.037

21. Dabaja BS, Suki D, Pro B, Bonnen M, Ajani J. Adenocarcinoma of the small bowel: presentation, prognostic factors, and outcome of 217 patients. Cancer. 2004; 101(3):518-26.

https://doi.org/10.1002/cncr.20404
22. Cimpeanu E, Zafar W, Circiumaru I, Prozumenshikov A, Salman S. Rare presentation of small bowel adenocarcinoma with neuroendocrine differentiation in the jejunum: A case report and summary of diagnostic and management options. Mol Clin Oncol. 2019;11(5):461-464. https://doi.org/10.3892/mco.2019.1925.

23. Coco D, Leanza S, Boccoli G. Small bowel adenocarcinoma (SBA) three years after colonic adenocarcinoma in an elderly patient: Case report in a National Institute of Health and Aging (INRCA) and review of the literature. Int J Surg Case Rep. 2014;5(12):939-43. https://doi.org/10.1016/j.ijscr.2014.07.011

24. Coco D, Leanza S, Boccoli G. Small bowel adenocarcinoma (SBA) three years after colonic adenocarcinoma in an elderly patient: Case report in a National Institute of Health and Aging (INRCA) and review of the literature. Int J Surg Case Rep. 2014;5(12):939-943. https://doi.org/10.1016/j.ijscr.2014.07.011. 